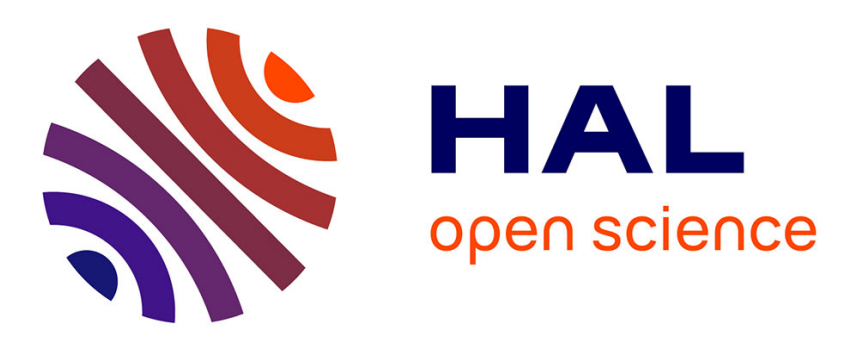

\title{
Asymptotic normality of the Parzen-Rosenblatt density estimator for strongly mixing random fields
}

\author{
Mohamed El Machkouri
}

\section{To cite this version:}

Mohamed El Machkouri. Asymptotic normality of the Parzen-Rosenblatt density estimator for strongly mixing random fields. 2010. hal-00530145

\section{HAL Id: hal-00530145 \\ https://hal.science/hal-00530145}

Preprint submitted on 27 Oct 2010

HAL is a multi-disciplinary open access archive for the deposit and dissemination of scientific research documents, whether they are published or not. The documents may come from teaching and research institutions in France or abroad, or from public or private research centers.
L'archive ouverte pluridisciplinaire HAL, est destinée au dépôt et à la diffusion de documents scientifiques de niveau recherche, publiés ou non, émanant des établissements d'enseignement et de recherche français ou étrangers, des laboratoires publics ou privés. 


\title{
Asymptotic normality of the Parzen-Rosenblatt density estimator for strongly mixing random fields
}

\author{
Mohamed EL MACHKOURI, \\ Laboratoire de Mathématiques Raphaël Salem \\ UMR CNRS 6085, Université de Rouen \\ mohamed.elmachkouri@univ-rouen.fr
}

October 6, 2010

\begin{abstract}
We prove the asymptotic normality of the kernel density estimator (introduced by Rosenblatt (1956) and Parzen (1962)) in the context of stationary strongly mixing random fields. Our approach is based on the Lindeberg's method rather than on Bernstein's small-block-large-block technique and coupling arguments widely used in previous works on nonparametric estimation for spatial processes. Our method allows us to consider only minimal conditions on the bandwidth parameter and provides a simple criterion on the strong mixing coefficients which do not depend on the bandwith.
\end{abstract}

AMS Subject Classifications (2000): 62G05, 62G07, 60G60.

Key words and phrases: Central limit theorem, kernel density estimator, strongly mixing random fields, spatial processes.

Short title: Kernel density estimation for random fields.

\section{Introduction and main result}

The kernel density estimator introduced by Rosenblatt [20] and Parzen [18] has received considerable attention in nonparametric estimation of probability densities for time series. If $\left(X_{i}\right)_{i \in \mathbb{Z}}$ is a stationary sequence of real random variables with a marginal density $f$ then the kernel density estimator of $f$ is defined for any positive integer $n$ 
and any $x$ in $\mathbb{R}$ by

$$
f_{n}(x)=\frac{1}{n b_{n}} \sum_{i=1}^{n} \mathrm{~K}\left(\frac{x-X_{i}}{b_{n}}\right)
$$

where $\mathrm{K}$ is a probability kernel and the bandwidth $b_{n}$ is a parameter which converges slowly to zero such that $n b_{n}$ goes to infinity (the bandwidth determines the amount of smoothness of the estimator). For small $b_{n}$ we get a very rough estimate and for large $b_{n}$ a smooth estimate. The literature dealing with the asymptotic properties of $f_{n}$ when the observations $\left(X_{i}\right)_{i \in \mathbb{Z}}$ are independent is very extensive (see Silverman [21]). In particular, Parzen [18] proved that when $\left(X_{i}\right)_{i \in \mathbb{Z}}$ is i.i.d. and the bandwidth $b_{n}$ goes to zero such that $n b_{n}$ goes to infinity then $\left(n b_{n}\right)^{1 / 2}\left(f_{n}\left(x_{0}\right)-\mathbb{E} f_{n}\left(x_{0}\right)\right)$ converges in distribution to the normal law with zero mean and variance $f\left(x_{0}\right) \int_{\mathbb{R}} \mathrm{K}^{2}(t) d t$. Under the same conditions on the bandwidth, this result was recently extended by $\mathrm{Wu}[24]$ for causal linear processes with i.i.d. innovations and by Dedecker and Merlevède [7] for strongly mixing sequences. Previously, Bosq, Merlevède and Peligrad [3] established a central limit theorem for the kernel density estimator $f_{n}$ when the sequence $\left(X_{i}\right)_{i \in \mathbb{Z}}$ is assumed to be strongly mixing but the bandwith parameter $b_{n}$ is assumed to satisfy $b_{n} \geq C n^{-1 / 3} \log n$ (for some positive constant $C$ ) which is stronger than the bandwith parameter assumption in [18], [7] and [24]. In this paper, we are going to establish Parzen's central limit theorem (see Theorem 1) for random variables which show spatial interaction (random fields). The problem is not trivial since $\mathbb{Z}^{d}$ does not have a natural ordering for $d \geq 2$. In particular, the martingale-difference method (Wu [24]) for time series seems to be difficult to apply for random fields. In order to establish our main result, we use the Lindeberg's method introduced in 1922 for the proof of the central limit theorem for independent random variables and successfully adapted in the spatial setting by Dedecker [6]. Our approach seems to be better than the Bernstein's smallblock-large-block technique and coupling arguments widely used in previous works on nonparametric estimation for spatial processes (see [4], [5], [12], [22]) since we are able to assume only minimal conditions on the bandwidth parameter and a simple criterion on the strong mixing coefficients which do not depend on the bandwith. Over the last few years nonparametric estimation for random fields (or spatial processes) was given increasing attention (see Guyon [11]). In fact, spatial data arise in various areas of research including econometrics, image analysis, meteorology and geostatistics. Some key references on nonparametric estimation for random fields are Biau [1], Carbon et al. [4], Carbon et al. [5], Hallin et al. [12], [13], Tran [22], Tran and Yakowitz [23] and Yao [25] who have investigated nonparametric density estimation for random fields and 
Biau and Cadre [2], El Machkouri [9], El Machkouri and Stoica [10], Hallin et al. [14] and Lu and Chen [15], [16] who have studied spatial prediction and spatial regression estimation.

Let $d$ be a positive integer and let $\left(X_{i}\right)_{i \in \mathbb{Z}^{d}}$ be a field of identically distributed real random variables with a marginal density $f$. Given two $\sigma$-algebras $\mathcal{U}$ and $\mathcal{V}$ of $\mathcal{F}$, different measures of their dependence have been considered in the literature. We are interested by one of them. The $\alpha$-mixing coefficient has been introduced by Rosenblatt [20] defined by

$$
\alpha(\mathcal{U}, \mathcal{V})=\sup \{|\mathbb{P}(A \cap B)-\mathbb{P}(A) \mathbb{P}(B)|, A \in \mathcal{U}, B \in \mathcal{V}\}
$$

In the sequel, we consider the strong mixing coefficients $\alpha_{1, \infty}(n)$ defined for each positive integer $n$ by

$$
\alpha_{1, \infty}(n)=\sup \left\{\alpha\left(\sigma\left(X_{k}\right), \mathcal{F}_{\Gamma}\right), k \in \mathbb{Z}^{d}, \Gamma \subset \mathbb{Z}^{d}, \rho(\Gamma,\{k\}) \geq n\right\}
$$

where $\mathcal{F}_{\Gamma}=\sigma\left(X_{i} ; i \in \Gamma\right)$ and the distance $\rho$ is defined for any subsets $\Gamma_{1}$ and $\Gamma_{2}$ of $\mathbb{Z}^{d}$ by $\rho\left(\Gamma_{1}, \Gamma_{2}\right)=\min \left\{|i-j|, i \in \Gamma_{1}, j \in \Gamma_{2}\right\}$ with $|i-j|=\max _{1 \leq s \leq d}\left|i_{s}-j_{s}\right|$ for any $i$ and $j$ in $\mathbb{Z}^{d}$. We say that the random field $\left(X_{i}\right)_{i \in \mathbb{Z}^{d}}$ is strongly mixing if $\lim _{n \rightarrow+\infty} \alpha_{1, \infty}(n)=0$. The class of mixing random fields in the above sense is very large (one can refer to Guyon [11] or Doukhan [8] for examples) and we recall that Dedecker [6] obtained a central limit theorem for the stationary random field $\left(X_{i}\right)_{i \in \mathbb{Z}^{d}}$ provided that $X_{0}$ has zero mean and finite variance and

$$
\sum_{k \in \mathbb{Z}^{d}} \int_{0}^{\alpha_{1, \infty}(|k|)} Q_{X_{0}}^{2}(u) d u<+\infty
$$

where $Q_{X_{0}}$ is the quantile function defined for any $u$ in $[0,1]$ by

$$
Q_{X_{0}}(u)=\inf \left\{t \geq 0 ; \mathbb{P}\left(\left|X_{0}\right|>t\right) \leq u\right\}
$$

We consider the density estimator of $f$ defined for any positive integer $n$ and any $x$ in $\mathbb{R}$ by

$$
f_{n}(x)=\frac{1}{n^{d} b_{n}} \sum_{i \in \Lambda_{n}} \mathrm{~K}\left(\frac{x-X_{i}}{b_{n}}\right)
$$

where $b_{n}$ is the bandwidth parameter, $\Lambda_{n}$ denotes the set $\{1, \ldots, n\}^{d}$ and $\mathrm{K}$ is a probability kernel. Our aim is to provide a sufficient condition on the strong mixing coefficients $\alpha_{1, \infty}(n)$ for $\left(n^{d} b_{n}\right)^{1 / 2}\left(f_{n}\left(x_{i}\right)-\mathbb{E} f_{n}\left(x_{i}\right)\right)_{1 \leq i \leq k},\left(x_{i}\right)_{1 \leq i \leq k} \in \mathbb{R}^{k}, k \in \mathbb{N}^{*}$, to converge in law 
to a multivariate normal distribution (Theorem 1) under minimal conditions on the bandwidths (that is $b_{n}$ goes to zero and $n^{d} b_{n}$ goes to infinity).

We consider the following assumptions:

(A1) The marginal probability distribution of each $X_{k}$ is absolutely continuous with continuous positive density function $f$.

(A2) The joint probability distribution of each $\left(X_{0}, X_{k}\right)$ is absolutely continuous with continuous joint density $f_{0, k}$.

(A3) $\mathrm{K}$ is a probability kernel with compact support and $\int_{\mathbb{R}} \mathrm{K}^{2}(u) d u<\infty$.

(A4) The bandwidth $b_{n}$ converges to zero and $n^{d} b_{n}$ goes to infinity.

Our main result is the following.

Theorem 1 Assume that (A1), (A2), (A3) and (A4) hold and

$$
\sum_{m=1}^{+\infty} m^{2 d-1} \alpha_{1, \infty}(m)<+\infty
$$

Then for any positive integer $k$ and any distinct points $x_{1}, \ldots, x_{k}$ in $\mathbb{R}$,

$$
\left(n^{d} b_{n}\right)^{1 / 2}\left(\begin{array}{c}
f_{n}\left(x_{1}\right)-\mathbb{E} f_{n}\left(x_{1}\right) \\
\vdots \\
f_{n}\left(x_{k}\right)-\mathbb{E} f_{n}\left(x_{k}\right)
\end{array}\right) \underset{n \rightarrow+\infty}{\stackrel{\mathcal{L}}{\longrightarrow}} \mathcal{N}(0, V)
$$

where $V$ is a diagonal matrix with diagonal elements $v_{i i}=f\left(x_{i}\right) \int_{\mathbb{R}} K^{2}(u) d u$.

Remark 1. A replacement of $\mathbb{E} f_{n}\left(x_{i}\right)$ by $f\left(x_{i}\right)$ for any $1 \leq i \leq k$ in (2) is a classical problem in density estimation theory. For example, if $f$ is assumed to be Lipschitz and if $\int_{\mathbb{R}}|u||\mathrm{K}(u)| d u<\infty$ then $\left|\mathbb{E} f_{n}\left(x_{i}\right)-f\left(x_{i}\right)\right|=O\left(b_{n}\right)$ and thus the centering $\mathbb{E} f_{n}\left(x_{i}\right)$ may be changed to $f\left(x_{i}\right)$ without affecting the above result provided that $n^{d} b_{n}^{3}$ converges to zero.

Remark 2. Theorem 1 is an extension of Theorem 3.1 by Bosq, Merlevède and Peligrad [3]. In fact, using a different approach, the authors obtained the same result for $d=1$ with an additional condition on the bandwith parameter (that is, there exists a positive constant $C$ such that $b_{n} \geq C n^{-1 / 3} \log n$ ). However, the condition (1) with $d=1$ is slightly more restrictive than the condition $\sum_{m>n} \alpha_{1, \infty}(m)=o\left(n^{-1}\right)$ obtained by Dedecker and Merlevède ([7], Corollary 4). We conjecture that Theorem 1 still holds under the condition $\sum_{m>n} m^{d-1} \alpha_{1, \infty}(m)=o\left(n^{-d}\right)$. 


\section{Proofs}

Proof of Theorem 1. Without loss of generality, we consider only the case $k=2$ and we refer to $x_{1}$ and $x_{2}$ as $x$ and $y(x \neq y)$. Let $\lambda_{1}$ and $\lambda_{2}$ be two constants such that $\lambda_{1}^{2}+\lambda_{2}^{2}=1$ and denote

$$
S_{n}=\lambda_{1}\left(n^{d} b_{n}\right)^{1 / 2}\left(f_{n}(x)-\mathbb{E} f_{n}(x)\right)+\lambda_{2}\left(n^{d} b_{n}\right)^{1 / 2}\left(f_{n}(y)-\mathbb{E} f_{n}(y)\right)=\sum_{i \in \Lambda_{n}} \frac{\Delta_{i}}{n^{d / 2}}
$$

where $\Delta_{i}=\lambda_{1} Z_{i}(x)+\lambda_{2} Z_{i}(y)$ and for any $z$ in $\mathbb{R}$,

$$
Z_{i}(z)=\frac{1}{\sqrt{b_{n}}}\left(\mathrm{~K}\left(\frac{z-X_{i}}{b_{n}}\right)-\mathbb{E K}\left(\frac{z-X_{i}}{b_{n}}\right)\right) .
$$

We consider the notations

$$
\eta=\left(\lambda_{1}^{2} f(x)+\lambda_{2}^{2} f(y)\right) \sigma^{2} \quad \text { and } \quad \sigma^{2}=\int_{\mathbb{R}} \mathrm{K}^{2}(u) d u .
$$

The proof of the following technical result is postponed to the annex.

Lemma $1 \mathbb{E}\left(\Delta_{0}^{2}\right)$ converges to $\eta$ and $\mathbb{E}\left|\Delta_{0} \Delta_{i}\right|=O\left(b_{n}\right)$ for any $i$ in $\mathbb{Z}^{d} \backslash\{0\}$.

In order to prove the convergence in distribution of $S_{n}$ to $\sqrt{\eta} \tau_{0}$ where $\tau_{0} \sim \mathcal{N}(0,1)$, we are going to follow the Lindeberg's method used in the proof of the central limit theorem for stationary random fields by Dedecker [6]. Let us note that several previous asymptotic results for kernel density estimates in the context of spatial processes were established using the so-called Bernstein's small-block-large-block technique and coupling arguments which lead to restrictive conditions on the bandwith parameter (see for example [4], [5], [12], [22]). Our approach seems to be better since we obtain a central limit theorem when the bandwith satisfies only Assumption (A4).

Let $\mu$ be the law of the stationary real random field $\left(X_{k}\right)_{k \in \mathbb{Z}^{d}}$ and consider the projection $\pi_{0}$ from $\mathbb{R}^{\mathbb{Z}^{d}}$ to $\mathbb{R}$ defined by $\pi_{0}(\omega)=\omega_{0}$ and the family of translation operators $\left(T^{k}\right)_{k \in \mathbb{Z}^{d}}$ from $\mathbb{R}^{\mathbb{Z}^{d}}$ to $\mathbb{R}^{\mathbb{Z}^{d}}$ defined by $\left(T^{k}(\omega)\right)_{i}=\omega_{i+k}$ for any $k \in \mathbb{Z}^{d}$ and any $\omega$ in $\mathbb{R}^{\mathbb{Z}^{d}}$. Denote by $\mathcal{B}$ the Borel $\sigma$-algebra of $\mathbb{R}$. The random field $\left(\pi_{0} \circ T^{k}\right)_{k \in \mathbb{Z}^{d}}$ defined on the probability space $\left(\mathbb{R}^{\mathbb{Z}^{d}}, \mathcal{B}^{\mathbb{Z}^{d}}, \mu\right)$ is stationary with the same law as $\left(X_{k}\right)_{k \in \mathbb{Z}^{d}}$, hence, without loss of generality, one can suppose that $(\Omega, \mathcal{F}, \mathbb{P})=\left(\mathbb{R}^{\mathbb{Z}^{d}}, \mathcal{B}^{\mathbb{Z}^{d}}, \mu\right)$ and $X_{k}=\pi_{0} \circ T^{k}$. On the lattice $\mathbb{Z}^{d}$ we define the lexicographic order as follows: if $i=\left(i_{1}, \ldots, i_{d}\right)$ and $j=\left(j_{1}, \ldots, j_{d}\right)$ are distinct elements of $\mathbb{Z}^{d}$, the notation $i<_{\operatorname{lex}} j$ means that either 
$i_{1}<j_{1}$ or for some $p$ in $\{2,3, \ldots, d\}, i_{p}<j_{p}$ and $i_{q}=j_{q}$ for $1 \leq q<p$. Let the sets $\left\{V_{i}^{M} ; i \in \mathbb{Z}^{d}, M \in \mathbb{N}^{*}\right\}$ be defined as follows:

$$
V_{i}^{1}=\left\{j \in \mathbb{Z}^{d} ; j<_{\text {lex }} i\right\}
$$

and for $M \geq 2$

$$
V_{i}^{M}=V_{i}^{1} \cap\left\{j \in \mathbb{Z}^{d} ;|i-j| \geq M\right\} \quad \text { where } \quad|i-j|=\max _{1 \leq l \leq d}\left|i_{l}-j_{l}\right| .
$$

For any subset $\Gamma$ of $\mathbb{Z}^{d}$ define $\mathcal{F}_{\Gamma}=\sigma\left(X_{i} ; i \in \Gamma\right)$ and set

$$
\mathbb{E}_{M}\left(X_{i}\right)=\mathbb{E}\left(X_{i} \mid \mathcal{F}_{V_{i}^{M}}\right), \quad M \in \mathbb{N}^{*}
$$

Let $g$ be a one to one map from $[1, M] \cap \mathbb{N}^{*}$ to a finite subset of $\mathbb{Z}^{d}$ and $\left(\xi_{i}\right)_{i \in \mathbb{Z}^{d}}$ a real random field. For all integers $k$ in $[1, M]$, we denote

$$
S_{g(k)}(\xi)=\sum_{i=1}^{k} \xi_{g(i)} \quad \text { and } \quad S_{g(k)}^{c}(\xi)=\sum_{i=k}^{M} \xi_{g(i)}
$$

with the convention $S_{g(0)}(\xi)=S_{g(M+1)}^{c}(\xi)=0$. To describe the set $\Lambda_{n}=\{1, \ldots, n\}^{d}$, we define the one to one map $g$ from $\left[1, n^{d}\right] \cap \mathbb{N}^{*}$ to $\Lambda_{n}$ by: $g$ is the unique function such that $g(k)<_{\operatorname{lex}} g(l)$ for $1 \leq k<l \leq n^{d}$. From now on, we consider a field $\left(\tau_{i}\right)_{i \in \mathbb{Z}^{d}}$ of i.i.d. random variables independent of $\left(X_{i}\right)_{i \in \mathbb{Z}^{d}}$ such that $\tau_{0}$ has the standard normal law $\mathcal{N}(0,1)$. We introduce the fields $Y$ and $\gamma$ defined for any $i$ in $\mathbb{Z}^{d}$ by

$$
Y_{i}=\frac{\Delta_{i}}{n^{d / 2}} \quad \text { and } \quad \gamma_{i}=\frac{\tau_{i} \sqrt{\eta}}{n^{d / 2}}
$$

where $\eta$ is defined by (3).

Let $h$ be any function from $\mathbb{R}$ to $\mathbb{R}$. For $0 \leq k \leq l \leq n^{d}+1$, we introduce $h_{k, l}(Y)=h\left(S_{g(k)}(Y)+S_{g(l)}^{c}(\gamma)\right)$. With the above convention we have that $h_{k, n^{d}+1}(Y)=$ $h\left(S_{g(k)}(Y)\right)$ and also $h_{0, l}(Y)=h\left(S_{g(l)}^{c}(\gamma)\right)$. In the sequel, we will often write $h_{k, l}$ instead of $h_{k, l}(Y)$. We denote by $B_{1}^{4}(\mathbb{R})$ the unit ball of $C_{b}^{4}(\mathbb{R}): h$ belongs to $B_{1}^{4}(\mathbb{R})$ if and only if it belongs to $C^{4}(\mathbb{R})$ and satisfies $\max _{0 \leq i \leq 4}\left\|h^{(i)}\right\|_{\infty} \leq 1$.

It suffices to prove that for all $h$ in $B_{1}^{4}(\mathbb{R})$,

$$
\mathbb{E}\left(h\left(S_{g\left(n^{d}\right)}(Y)\right)\right) \underset{n \rightarrow+\infty}{\longrightarrow} \mathbb{E}\left(h\left(\tau_{0} \sqrt{\eta}\right)\right) .
$$


We use Lindeberg's decomposition:

$$
\mathbb{E}\left(h\left(S_{g\left(n^{d}\right)}(Y)\right)-h\left(\tau_{0} \sqrt{\eta}\right)\right)=\sum_{k=1}^{n^{d}} \mathbb{E}\left(h_{k, k+1}-h_{k-1, k}\right) .
$$

Now,

$$
h_{k, k+1}-h_{k-1, k}=h_{k, k+1}-h_{k-1, k+1}+h_{k-1, k+1}-h_{k-1, k} .
$$

Applying Taylor's formula we get that:

$$
h_{k, k+1}-h_{k-1, k+1}=Y_{g(k)} h_{k-1, k+1}^{\prime}+\frac{1}{2} Y_{g(k)}^{2} h_{k-1, k+1}^{\prime \prime}+R_{k}
$$

and

$$
h_{k-1, k+1}-h_{k-1, k}=-\gamma_{g(k)} h_{k-1, k+1}^{\prime}-\frac{1}{2} \gamma_{g(k)}^{2} h_{k-1, k+1}^{\prime \prime}+r_{k}
$$

where $\left|R_{k}\right| \leq Y_{g(k)}^{2}\left(1 \wedge\left|Y_{g(k)}\right|\right)$ and $\left|r_{k}\right| \leq \gamma_{g(k)}^{2}\left(1 \wedge\left|\gamma_{g(k)}\right|\right)$. Since $\left(Y, \tau_{i}\right)_{i \neq g(k)}$ is independent of $\tau_{g(k)}$, it follows that

$$
\mathbb{E}\left(\gamma_{g(k)} h_{k-1, k+1}^{\prime}\right)=0 \quad \text { and } \quad \mathbb{E}\left(\gamma_{g(k)}^{2} h_{k-1, k+1}^{\prime \prime}\right)=\mathbb{E}\left(\frac{\eta}{n^{d}} h_{k-1, k+1}^{\prime \prime}\right)
$$

Hence, we obtain

$$
\begin{aligned}
\mathbb{E}\left(h\left(S_{g\left(n^{d}\right)}(Y)\right)-h\left(\tau_{0} \sqrt{\eta}\right)\right)= & \sum_{k=1}^{n^{d}} \mathbb{E}\left(Y_{g(k)} h_{k-1, k+1}^{\prime}\right) \\
& +\sum_{k=1}^{n^{d}} \mathbb{E}\left(\left(Y_{g(k)}^{2}-\frac{\eta}{n^{d}}\right) \frac{h_{k-1, k+1}^{\prime \prime}}{2}\right) \\
& +\sum_{k=1}^{n^{d}} \mathbb{E}\left(R_{k}+r_{k}\right) .
\end{aligned}
$$

Let $1 \leq k \leq n^{d}$ be fixed. Noting that $\Delta_{0}$ is bounded by $4\|\mathrm{~K}\|_{\infty} / \sqrt{b_{n}}$ and applying Lemma 1, we derive

$$
\mathbb{E}\left|R_{k}\right| \leq \frac{\mathbb{E}\left|\Delta_{0}\right|^{3}}{n^{3 d / 2}}=O\left(\frac{1}{\left(n^{3 d} b_{n}\right)^{1 / 2}}\right)
$$

and

$$
\mathbb{E}\left|r_{k}\right| \leq \frac{\mathbb{E}\left|\gamma_{0}\right|^{3}}{n^{3 d / 2}} \leq \frac{\eta^{3 / 2} \mathbb{E}\left|\tau_{0}\right|^{3}}{n^{3 d / 2}}=O\left(\frac{1}{n^{3 d / 2}}\right)
$$

Consequently, we obtain

$$
\sum_{k=1}^{n^{d}} \mathbb{E}\left(\left|R_{k}\right|+\left|r_{k}\right|\right)=O\left(\frac{1}{\left(n^{d} b_{n}\right)^{1 / 2}}+\frac{1}{n^{d / 2}}\right)=o(1) .
$$


Now, it is sufficient to show

$$
\lim _{n \rightarrow+\infty} \sum_{k=1}^{n^{d}}\left(\mathbb{E}\left(Y_{g(k)} h_{k-1, k+1}^{\prime}\right)+\mathbb{E}\left(\left(Y_{g(k)}^{2}-\frac{\eta}{n^{d}}\right) \frac{h_{k-1, k+1}^{\prime \prime}}{2}\right)\right)=0 .
$$

First, we focus on $\sum_{k=1}^{n^{d}} \mathbb{E}\left(Y_{g(k)} h_{k-1, k+1}^{\prime}\right)$. For all $M$ in $\mathbb{N}^{*}$ and all integer $k$ in $\left[1, n^{d}\right]$, we define

$$
E_{k}^{M}=g\left([1, k] \cap \mathbb{N}^{*}\right) \cap V_{g(k)}^{M} \quad \text { and } \quad S_{g(k)}^{M}(Y)=\sum_{i \in E_{k}^{M}} Y_{i} .
$$

For any function $\Psi$ from $\mathbb{R}$ to $\mathbb{R}$, we define $\Psi_{k-1, l}^{M}=\Psi\left(S_{g(k)}^{M}(Y)+S_{g(l)}^{c}(\gamma)\right.$ ) (we are going to apply this notation to the successive derivatives of the function $h$ ).

For any integer $n$, we define

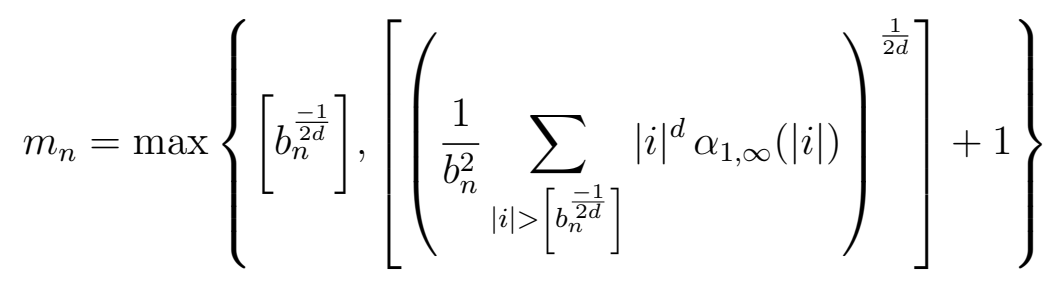

where [.] denotes the integer part function. The following technical lemma is the spatial version of a result by Bosq, Merlevède and Peligrad ([3], pages 88-89). In order to be self-contained, the proof is done in the appendix.

Lemma 2 Under Assumption (A4) and the mixing condition (1), we have

$$
m_{n}^{d} \rightarrow \infty, \quad m_{n}^{d} b_{n} \rightarrow 0 \quad \text { and } \quad \frac{1}{m_{n}^{d} b_{n}} \sum_{|i|>m_{n}}|i|^{d} \alpha_{1, \infty}(|i|) \rightarrow 0 .
$$

Our aim is to show that

$$
\lim _{n \rightarrow+\infty} \sum_{k=1}^{n^{d}} \mathbb{E}\left(Y_{g(k)} h_{k-1, k+1}^{\prime}-Y_{g(k)}\left(S_{g(k-1)}(Y)-S_{g(k)}^{m_{n}}(Y)\right) h_{k-1, k+1}^{\prime \prime}\right)=0 .
$$

First, we use the decomposition

$$
Y_{g(k)} h_{k-1, k+1}^{\prime}=Y_{g(k)} h_{k-1, k+1}^{\prime m_{n}}+Y_{g(k)}\left(h_{k-1, k+1}^{\prime}-h_{k-1, k+1}^{\prime m_{n}}\right) .
$$

We consider a one to one map $m$ from $\left[1,\left|E_{k}^{m_{n}}\right|\right] \cap \mathbb{N}^{*}$ to $E_{k}^{m_{n}}$ and such that $\mid m(i)$ $g(k)|\leq| m(i-1)-g(k) \mid$. This choice of $m$ ensures that $S_{m(i)}(Y)$ and $S_{m(i-1)}(Y)$ are

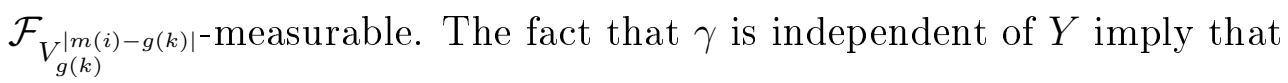

$$
\mathbb{E}\left(Y_{g(k)} h^{\prime}\left(S_{g(k+1)}^{c}(\gamma)\right)\right)=0
$$


Therefore

$$
\left|\mathbb{E}\left(Y_{g(k)} h_{k-1, k+1}^{\prime m_{n}}\right)\right|=\left|\sum_{i=1}^{\left|E_{k}^{m_{n}}\right|} \mathbb{E}\left(Y_{g(k)}\left(\theta_{i}-\theta_{i-1}\right)\right)\right|
$$

where $\theta_{i}=h^{\prime}\left(S_{m(i)}(Y)+S_{g(k+1)}^{c}(\gamma)\right)$.

Since $S_{m(i)}(Y)$ and $S_{m(i-1)}(Y)$ are $\mathcal{F}_{V_{g(k)}^{|m(i)-g(k)|} \text {-measurable, we can take the conditional }}$ expectation of $Y_{g(k)}$ with respect to $\mathcal{F}_{V_{g(k)}^{|m(i)-g(k)|}}$ in the right hand side of (6). On the other hand the function $h^{\prime}$ is 1-Lipschitz, hence

$$
\left|\theta_{i}-\theta_{i-1}\right| \leq\left|Y_{m(i)}\right|
$$

Consequently,

$$
\left|\mathbb{E}\left(Y_{g(k)}\left(\theta_{i}-\theta_{i-1}\right)\right)\right| \leq \mathbb{E}\left|Y_{m(i)} \mathbb{E}_{|m(i)-g(k)|}\left(Y_{g(k)}\right)\right|
$$

and

$$
\left|\mathbb{E}\left(Y_{g(k)} h_{k-1, k+1}^{\prime m_{n}}\right)\right| \leq \sum_{i=1}^{\left|E_{k}^{m_{n}}\right|} \mathbb{E}\left|Y_{m(i)} \mathbb{E}_{|m(i)-g(k)|}\left(Y_{g(k)}\right)\right|
$$

Hence,

$$
\begin{aligned}
\left|\sum_{k=1}^{n^{d}} \mathbb{E}\left(Y_{g(k)} h_{k-1, k+1}^{\prime m_{n}}\right)\right| & \leq \frac{1}{n^{d}} \sum_{k=1}^{n^{d}} \sum_{i=1}^{\left|E_{k}^{m_{n}}\right|} \mathbb{E}\left|\Delta_{m(i)} \mathbb{E}_{|m(i)-g(k)|}\left(\Delta_{g(k)}\right)\right| \\
& \leq \sum_{|j| \geq m_{n}}\left\|\Delta_{j} \mathbb{E}_{|j|}\left(\Delta_{0}\right)\right\|_{1} .
\end{aligned}
$$

For any $j$ in $\mathbb{Z}^{d}$, we have

$$
\left\|\Delta_{j} \mathbb{E}_{|j|}\left(\Delta_{0}\right)\right\|_{1}=\operatorname{Cov}\left(\left|\Delta_{j}\right|\left(\mathbb{I}_{\mathbb{E}_{|j|}\left(\Delta_{0}\right) \geq 0}-\mathbb{I}_{\mathbb{E}_{|j|}\left(\Delta_{0}\right)<0}\right), \Delta_{0}\right) .
$$

So, applying Rio's covariance inequality (cf. [19], Theorem 1.1), we obtain

$$
\left\|\Delta_{j} \mathbb{E}_{|j|}\left(\Delta_{0}\right)\right\|_{1} \leq 4 \int_{0}^{\alpha_{1, \infty}(|j|)} Q_{\Delta_{0}}^{2}(u) d u
$$

where $Q_{\Delta_{0}}$ is defined by $Q_{\Delta_{0}}(u)=\inf \left\{t \geq 0 ; \mathbb{P}\left(\left|\Delta_{0}\right|>t\right) \leq u\right\}$ for any $u$ in $[0,1]$. Since $\Delta_{0}$ is bounded by $4\|\mathrm{~K}\|_{\infty} / \sqrt{b_{n}}$, we have

$$
Q_{\Delta_{0}}(u) \leq \frac{4\|\mathrm{~K}\|_{\infty}}{\sqrt{b_{n}}} \quad \text { and } \quad\left\|\Delta_{j} \mathbb{E}_{|j|}\left(\Delta_{0}\right)\right\|_{1} \leq \frac{64\|\mathrm{~K}\|_{\infty}^{2}}{b_{n}} \alpha_{1, \infty}(|j|) .
$$


Finally, we derive

$$
\begin{aligned}
\left|\sum_{k=1}^{n^{d}} \mathbb{E}\left(Y_{g(k)} h_{k-1, k+1}^{\prime m_{n}}\right)\right| & \leq \frac{64\|K\|_{\infty}^{2}}{b_{n}} \sum_{|j| \geq m_{n}} \alpha_{1, \infty}(|j|) \\
& \leq \frac{64\|K\|_{\infty}^{2}}{m_{n}^{d} b_{n}} \sum_{|j| \geq m_{n}}|j|^{d} \alpha_{1, \infty}(|j|) \\
& =o(1) \quad \text { by }(5) .
\end{aligned}
$$

Applying again Taylor's formula, it remains to consider

$$
Y_{g(k)}\left(h_{k-1, k+1}^{\prime}-h_{k-1, k+1}^{\prime m_{n}}\right)=Y_{g(k)}\left(S_{g(k-1)}(Y)-S_{g(k)}^{m_{n}}(Y)\right) h_{k-1, k+1}^{\prime \prime}+R_{k}^{\prime},
$$

where $\left|R_{k}^{\prime}\right| \leq 2\left|Y_{g(k)}\left(S_{g(k-1)}(Y)-S_{g(k)}^{m_{n}}(Y)\right)\left(1 \wedge\left|S_{g(k-1)}(Y)-S_{g(k)}^{m_{n}}(Y)\right|\right)\right|$. Denoting $W_{n}=\left\{-m_{n}+1, \ldots, m_{n}-1\right\}^{d}$ and $W_{n}^{*}=W_{n} \backslash\{0\}$, it follows that

$$
\begin{aligned}
& \sum_{k=1}^{n^{d}} \mathbb{E}\left|R_{k}^{\prime}\right| \leq 2 \mathbb{E}\left(\left|\Delta_{0}\right|\left(\sum_{i \in W_{n}}\left|\Delta_{i}\right|\right)\left(1 \wedge \frac{1}{n^{d / 2}} \sum_{i \in W_{n}}\left|\Delta_{i}\right|\right)\right) \\
& =2 \mathbb{E}\left(\left(\Delta_{0}^{2}+\sum_{i \in W_{n}^{*}}\left|\Delta_{0} \Delta_{i}\right|\right)\left(1 \wedge \frac{1}{n^{d / 2}} \sum_{i \in W_{n}}\left|\Delta_{i}\right|\right)\right) \\
& \leq \frac{2}{n^{d / 2}} \sum_{i \in W_{n}} \mathbb{E}\left(\Delta_{0}^{2}\left|\Delta_{i}\right|\right)+2 \sum_{i \in W_{n}^{*}} \mathbb{E}\left|\Delta_{0} \Delta_{i}\right| \\
& \leq \frac{8\|\mathrm{~K}\|_{\infty}}{\left(n^{d} b_{n}\right)^{1 / 2}} \sum_{i \in W_{n}} \mathbb{E}\left(\left|\Delta_{0} \Delta_{i}\right|\right)+2 \sum_{i \in W_{n}^{*}} \mathbb{E}\left|\Delta_{0} \Delta_{i}\right| \quad \text { since } \Delta_{0} \leq \frac{4\|K\|_{\infty}}{\sqrt{b_{n}}} \text { a.s. } \\
& =\frac{8 \mathbb{E}\left(\Delta_{0}^{2}\right)\|\mathrm{K}\|_{\infty}}{\left(n^{d} b_{n}\right)^{1 / 2}}+2\left(1+\frac{4\|\mathrm{~K}\|_{\infty}}{\left(n^{d} b_{n}\right)^{1 / 2}}\right) \sum_{i \in W_{n}^{*}} \mathbb{E}\left(\left|\Delta_{0} \Delta_{i}\right|\right) \\
& =O\left(\frac{1}{\left(n^{d} b_{n}\right)^{1 / 2}}+m_{n}^{d} b_{n}\left(1+\frac{1}{\left(n^{d} b_{n}\right)^{1 / 2}}\right)\right) \quad(\text { by Lemma } 1) \\
& =o(1) \text { by }(5) \text {. }
\end{aligned}
$$

So, we have shown that

$$
\lim _{n \rightarrow+\infty} \sum_{k=1}^{n^{d}} \mathbb{E}\left(Y_{g(k)} h_{k-1, k+1}^{\prime}-Y_{g(k)}\left(S_{g(k-1)}-S_{g(k)}^{m_{n}}\right) h_{k-1, k+1}^{\prime \prime}\right)=0 .
$$

In order to obtain (4) it remains to control

$$
F_{0}=\mathbb{E}\left(\sum_{k=1}^{n^{d}} h_{k-1, k+1}^{\prime \prime}\left(\frac{Y_{g(k)}^{2}}{2}+Y_{g(k)}\left(S_{g(k-1)}(Y)-S_{g(k)}^{m_{n}}(Y)\right)-\frac{\eta}{2 n^{d}}\right)\right) .
$$


We consider the following sets:

$$
\Lambda_{n}^{m_{n}}=\left\{i \in \Lambda_{n} ; \rho\left(\{i\}, \partial \Lambda_{n}\right) \geq m_{n}\right\} \quad \text { and } \quad I_{n}^{m_{n}}=\left\{1 \leq i \leq n^{d} ; g(i) \in \Lambda_{n}^{m_{n}}\right\} \text {, }
$$

and the function $\Psi$ from $\mathbb{R}^{\mathbb{Z}^{d}}$ to $\mathbb{R}$ such that

$$
\Psi(\Delta)=\Delta_{0}^{2}+\sum_{i \in V_{0}^{1} \cap W_{n}} 2 \Delta_{0} \Delta_{i} \quad \text { where } W_{n}=\left\{-m_{n}+1, \ldots, m_{n}-1\right\}^{d} .
$$

For $1 \leq k \leq n^{d}$, we set $D_{k}^{(n)}=\eta-\Psi \circ T^{g(k)}(\Delta)$. By definition of $\Psi$ and of the set $I_{n}^{m_{n}}$, we have for any $k$ in $I_{n}^{m_{n}}$

$$
\Psi \circ T^{g(k)}(\Delta)=\Delta_{g(k)}^{2}+2 \Delta_{g(k)}\left(S_{g(k-1)}(\Delta)-S_{g(k)}^{m_{n}}(\Delta)\right) .
$$

Therefore for $k$ in $I_{n}^{m_{n}}$

$$
\frac{D_{k}^{(n)}}{n^{d}}=\frac{\eta}{n^{d}}-Y_{g(k)}^{2}-2 Y_{g(k)}\left(S_{g(k-1)}(Y)-S_{g(k)}^{m_{n}}(Y)\right) .
$$

Since $\lim _{n \rightarrow+\infty} n^{-d}\left|I_{n}^{m_{n}}\right|=1$, it remains to consider

$$
F_{1}=\left|\mathbb{E}\left(\frac{1}{n^{d}} \sum_{k=1}^{n^{d}} h_{k-1, k+1}^{\prime \prime} D_{k}^{(n)}\right)\right|
$$

Applying Lemma 1, we have

$$
\begin{aligned}
F_{1} & \leq\left|\mathbb{E}\left(\frac{1}{n^{d}} \sum_{k=1}^{n^{d}} h_{k-1, k+1}^{\prime \prime}\left(\Delta_{g(k)}^{2}-\mathbb{E}\left(\Delta_{0}^{2}\right)\right)\right)\right|+\left|\eta-\mathbb{E}\left(\Delta_{0}^{2}\right)\right|+2 \sum_{j \in V_{0}^{1} \cap W_{n}} \mathbb{E}\left|\Delta_{0} \Delta_{j}\right| \\
& \leq\left|\mathbb{E}\left(\frac{1}{n^{d}} \sum_{k=1}^{n^{d}} h_{k-1, k+1}^{\prime \prime}\left(\Delta_{g(k)}^{2}-\mathbb{E}\left(\Delta_{0}^{2}\right)\right)\right)\right|+o(1)+O\left(m_{n}^{d} b_{n}\right),
\end{aligned}
$$

it suffices to prove that

$$
F_{2}=\left|\mathbb{E}\left(\frac{1}{n^{d}} \sum_{k=1}^{n^{d}} h_{k-1, k+1}^{\prime \prime}\left(\Delta_{g(k)}^{2}-\mathbb{E}\left(\Delta_{0}^{2}\right)\right)\right)\right|
$$

goes to zero as $n$ goes to infinity. Let $M>0$ be fixed. We have $F_{2} \leq F_{2}^{\prime}+F_{2}^{\prime \prime}$ where

$$
F_{2}^{\prime}=\left|\mathbb{E}\left(\frac{1}{n^{d}} \sum_{k=1}^{n^{d}} h_{k-1, k+1}^{\prime \prime}\left(\Delta_{g(k)}^{2}-\mathbb{E}_{M}\left(\Delta_{g(k)}^{2}\right)\right)\right)\right|
$$


and

$$
F_{2}^{\prime \prime}=\left|\mathbb{E}\left(\frac{1}{n^{d}} \sum_{k=1}^{n^{d}} h_{k-1, k+1}^{\prime \prime}\left(\mathbb{E}_{M}\left(\Delta_{g(k)}^{2}\right)-\mathbb{E}\left(\Delta_{0}^{2}\right)\right)\right)\right|
$$

where we recall the notation $\mathbb{E}_{M}\left(\Delta_{g(k)}^{2}\right)=\mathbb{E}\left(\Delta_{g(k)}^{2} \mid \mathcal{F}_{V_{g(k)}^{M}}\right)$. The following result is a Serfling type inequality which can be found in [17].

Lemma 3 Let $\mathcal{U}$ and $\mathcal{V}$ be two $\sigma$-algebras and let $X$ be a random variable measurable with respect to $\mathcal{U}$. If $1 \leq p \leq r \leq \infty$ then

$$
\|\mathbb{E}(X \mid \mathcal{V})-\mathbb{E}(X)\|_{p} \leq 2\left(2^{1 / p}+1\right)(\alpha(\mathcal{U}, \mathcal{V}))^{\frac{1}{p}-\frac{1}{r}}\|X\|_{r}
$$

Applying Lemma 3 and keeping in mind that $\Delta_{0}$ is bounded by $4\|\mathrm{~K}\|_{\infty} / \sqrt{b_{n}}$, we derive

$$
F_{2}^{\prime \prime} \leq\left\|\mathbb{E}_{M}\left(\Delta_{0}^{2}\right)-\mathbb{E}\left(\Delta_{0}^{2}\right)\right\|_{1} \leq \frac{96\|\mathrm{~K}\|_{\infty}^{2}}{b_{n}} \alpha_{1, \infty}(M)
$$

In the other part,

$$
F_{2}^{\prime} \leq \frac{1}{n^{d}} \sum_{k=1}^{n^{d}}\left(J_{k}^{1}(M)+J_{k}^{2}(M)\right)
$$

where

$$
J_{k}^{1}(M)=\left|\mathbb{E}\left(h_{k-1, k+1}^{\prime \prime M} \circ T^{-g(k)}\left(\Delta_{0}^{2}-\mathbb{E}_{M}\left(\Delta_{0}^{2}\right)\right)\right)\right|=0
$$

since $h_{k-1, k+1}^{\prime \prime M} \circ T^{-g(k)}$ is $\mathcal{F}_{V_{0}^{M}}$-measurable and

$$
\begin{aligned}
J_{k}^{2}(M) & =\left|\mathbb{E}\left(\left(h_{k-1, k+1}^{\prime \prime} \circ T^{-g(k)}-h_{k-1, k+1}^{\prime \prime M} \circ T^{-g(k)}\right)\left(\Delta_{0}^{2}-\mathbb{E}_{M}\left(\Delta_{0}^{2}\right)\right)\right)\right| \\
& \leq \mathbb{E}\left(\left(2 \wedge \sum_{|i|<M} \frac{\left|\Delta_{i}\right|}{n^{d / 2}}\right) \Delta_{0}^{2}\right) \\
& \leq \frac{4\|\mathrm{~K}\|_{\infty} \mathbb{E}\left(\Delta_{0}^{2}\right)}{\left(n^{d} b_{n}\right)^{1 / 2}}+\frac{4\|\mathrm{~K}\|_{\infty}}{\left(n^{d} b_{n}\right)^{1 / 2}} \sum_{\substack{|i|<M \\
i \neq 0}} \mathbb{E}\left|\Delta_{i} \Delta_{0}\right| \quad \text { since } \Delta_{0} \leq \frac{4\|K\|_{\infty}}{\sqrt{b_{n}}} \text { a.s. } \\
& =O\left(\frac{1}{\left(n^{d} b_{n}\right)^{1 / 2}}+\frac{M^{d} \sqrt{b_{n}}}{n^{d / 2}}\right) \quad \text { (by Lemma 1) }
\end{aligned}
$$

So, putting $M=b_{n}^{\frac{-1}{2 d-1}}$ and keeping in mind that $\sum_{m \geq 0} m^{2 d-1} \alpha_{1, \infty}(m)<+\infty$, we derive

$$
F_{2}=O\left(M^{2 d-1} \alpha_{1, \infty}(M)\right)+O\left(\frac{1+b_{n}^{\frac{d-1}{2 d-1}}}{\left(n^{d} b_{n}\right)^{1 / 2}}\right)=o(1) .
$$

The proof of Theorem 1 is complete. 


\section{Appendix}

Proof of Lemma 1. For any $i$ in $\mathbb{Z}^{d}$ and any $z$ in $\mathbb{R}$, we note $\mathrm{K}_{i}(z)=\mathrm{K}\left(\frac{z-X_{i}}{b_{n}}\right)$. So, if $s$ and $t$ belongs to $\mathbb{R}$, we have

$$
\mathbb{E}\left(Z_{0}(s) Z_{0}(t)\right)=\frac{1}{b_{n}}\left(\mathbb{E}\left(\mathrm{K}_{0}(s) \mathrm{K}_{0}(t)\right)-\mathbb{E} \mathrm{K}_{0}(s) \mathbb{E} \mathrm{K}_{0}(t)\right)
$$

and

$$
\lim _{n \rightarrow+\infty} \frac{1}{b_{n}} \mathbb{E}\left(\mathrm{K}_{0}(s) \mathrm{K}_{0}(t)\right)=\lim _{n \rightarrow+\infty} \int_{\mathbb{R}} \mathrm{K}(v) \mathrm{K}\left(v+\frac{t-s}{b_{n}}\right) f\left(s-v b_{n}\right) d v=\delta_{s t} f(s) \sigma^{2}
$$

where $\delta_{s t}=1$ if $s=t$ and $\delta_{s t}=0$ if $s \neq t$. We have also

$$
\lim _{n \rightarrow+\infty} \frac{1}{b_{n}} \mathbb{E} K_{0}(s) \mathbb{E} K_{0}(t)=\lim _{n \rightarrow+\infty} b_{n} \int_{\mathbb{R}} \mathrm{K}(v) f\left(s-v b_{n}\right) d v \int_{\mathbb{R}} \mathrm{K}(w) f\left(t-w b_{n}\right) d w=0 .
$$

So, we obtain

$$
\mathbb{E}\left(\Delta_{0}^{2}\right)=\lambda_{1}^{2} \mathbb{E}\left(Z_{0}^{2}(x)\right)+\lambda_{2}^{2} \mathbb{E}\left(Z_{0}^{2}(y)\right)+2 \lambda_{1} \lambda_{2} \mathbb{E}\left(Z_{0}(x) Z_{0}(y)\right) \underset{n \rightarrow+\infty}{\longrightarrow} \eta .
$$

Let $i \neq 0$ be fixed in $\mathbb{Z}^{d}$. We have

$\mathbb{E}\left|\Delta_{0} \Delta_{i}\right| \leq \lambda_{1}^{2} \mathbb{E}\left|Z_{0}(x) Z_{i}(x)\right|+\lambda_{2}^{2} \mathbb{E}\left|Z_{0}(y) Z_{i}(y)\right|+\lambda_{1} \lambda_{2} \mathbb{E}\left|Z_{0}(x) Z_{i}(y)\right|+\lambda_{1} \lambda_{2} \mathbb{E}\left|Z_{0}(y) Z_{i}(x)\right|$.

For any $s$ and $t$ in $\mathbb{R}$,

$$
\mathbb{E}\left|Z_{0}(s) Z_{i}(t)\right| \leq \frac{1}{b_{n}} \mathbb{E}\left|\mathrm{K}_{0}(s) \mathrm{K}_{i}(t)\right|+\frac{1}{b_{n}} \mathbb{E}\left|\mathrm{K}_{0}(s)\right| \mathbb{E}\left|\mathrm{K}_{0}(t)\right| .
$$

Moreover, using Assumptions (A2) and (A3), we have

$$
\frac{1}{b_{n}} \mathbb{E}\left|\mathrm{K}_{0}(s)\right| \mathbb{E}\left|\mathrm{K}_{0}(t)\right|=b_{n} \int_{\mathbb{R}}|\mathrm{K}(u)| f\left(s-u b_{n}\right) d u \int_{\mathbb{R}}|\mathrm{K}(v)| f\left(t-v b_{n}\right) d v=O\left(b_{n}\right)
$$

and

$$
\frac{1}{b_{n}} \mathbb{E}\left|\mathrm{K}_{0}(s) \mathrm{K}_{i}(t)\right|=b_{n} \iint_{\mathbb{R}^{2}}\left|\mathrm{~K}\left(w_{1}\right) \mathrm{K}\left(w_{2}\right)\right| f_{0, i}\left(s-w_{1} b_{n}, t-w_{2} b_{n}\right) d w_{1} d w_{2}=O\left(b_{n}\right) .
$$

So, we obtain for any $s$ and $t$ in $\mathbb{R}$

$$
\mathbb{E}\left|Z_{0}(s) Z_{i}(t)\right|=O\left(b_{n}\right)
$$

The proof of Lemma 1 is completed by combining (7) and (8). 
Proof of Lemma 2. We follow the proof by Bosq, Merlevède and Peligrad ([3], pages 88-89). First, $m_{n}^{d}$ goes to infinity since $b_{n}$ goes to zero and $m_{n} \geq\left[b_{n}^{-\frac{1}{2 d}}\right]$. For any positive integer $m$, we consider

$$
\psi(m)=\sum_{|i|>m}|i|^{d} \alpha_{1, \infty}(|i|) .
$$

Since the mixing condition (1) is equivalent to $\sum_{k \in \mathbb{Z}^{d}}|k|^{d} \alpha_{1, \infty}(|k|)<\infty$, we know that $\psi(m)$ converges to zero as $m$ goes to infinity. Moreover, we have

$$
m_{n}^{d} b_{n} \leq \max \left\{\sqrt{b_{n}}, C_{d}\left(\sqrt{\psi\left(\left[b_{n}^{-\frac{1}{2 d}}\right]\right)}+2^{d} b_{n}\right)\right\} \underset{n \rightarrow+\infty}{\longrightarrow} 0
$$

where $C_{d}$ is some positive constant depending on the dimension $d$. We have also

$$
m_{n}^{d} \geq \frac{1}{b_{n}} \sqrt{\psi\left(\left[b_{n}^{-\frac{1}{2 d}}\right]\right)} \geq \frac{1}{b_{n}} \sqrt{\psi\left(m_{n}\right)} \quad \text { since }\left[b_{n}^{-\frac{1}{2 d}}\right] \leq m_{n} .
$$

Finally, we obtain

$$
\frac{1}{m_{n}^{d} b_{n}} \sum_{|i|>m_{n}}|i|^{d} \alpha_{1, \infty}(|i|) \leq \sqrt{\psi\left(m_{n}\right)} \underset{n \rightarrow+\infty}{\longrightarrow} 0 .
$$

The proof of Lemma 2 is complete.

\section{References}

[1] G. Biau. Spatial kernel density estimation. Math. Methods Statist., 12(4):371-390, 2003.

[2] G. Biau and B. Cadre. Nonparametric spatial prediction. Stat. Inference Stoch. Process., 7(3):327-349, 2004.

[3] D. Bosq, Merlevède F., and M. Peligrad. Asymptotic normality for density kernel estimators in discrete and continuous time. J. Multivariate Anal., 68:78-95, 1999.

[4] M. Carbon, M. Hallin, and L.T. Tran. Kernel density estimation for random fields: the $l_{1}$ theory. Journal of nonparametric Statistics, 6:157-170, 1996.

[5] M. Carbon, L.T. Tran, and B. Wu. Kernel density estimation for random fields. Statist. Probab. Lett., 36:115-125, 1997. 
[6] J. Dedecker. A central limit theorem for stationary random fields. Probab. Theory Relat. Fields, 110:397-426, 1998.

[7] J. Dedecker and F. Merlevède. Necessary and sufficient conditions for the conditional central limit theorem. Annals of Probability, 30(3):1044-1081, 2002.

[8] P. Doukhan. Mixing: properties and examples, volume 85. Lecture Notes in Statistics, Berlin, 1994.

[9] M. El Machkouri. Nonparametric regression estimation for random fields in a fixed-design. Stat. Inference Stoch. Process., 10(1):29-47, 2007.

[10] M. El Machkouri and R. S. Stoica. Asymptotic normality of kernel estimates in a regression model for random fields. To appear in Journal of Nonparametric Statistics, 2010.

[11] X. Guyon. Random fields on a Network: Modeling, Statistics and Applications. Springer, New York, 1995.

[12] M. Hallin, Z. Lu, and L.T. Tran. Density estimation for spatial linear processes. Bernoulli, 7:657-668, 2001.

[13] M. Hallin, Z. Lu, and L.T. Tran. Density estimation for spatial processes: the $l_{1}$ theory. J. Multivariate Anal., 88(1):61-75, 2004.

[14] M. Hallin, Z. Lu, and L.T. Tran. Local linear spatial regression. Ann. Statist., 32(6):2469-2500, 2004.

[15] Z. Lu and X. Chen. Spatial nonparametric regression estimation: Non-isotropic case. Acta Mathematicae Applicatae Sinica, English series, 18:641-656, 2002.

[16] Z. Lu and X. Chen. Spatial kernel regression estimation: weak consistency. Statist. Probab. Lett., 68:125-136, 2004.

[17] D. L. McLeish. A maximal inequality and dependent strong laws. Ann. Probab., $3(5): 829-839,1975$.

[18] E. Parzen. On the estimation of a probability density and the mode. Ann. Math. Statist., 33:1965-1976, 1962. 
[19] E. Rio. Covariance inequalities for strongly mixing processes. Ann. Inst. H. Poincaré Probab. Statist., 29(4):587-597, 1993.

[20] M. Rosenblatt. A central limit theorem and a strong mixing condition. Proc. Nat. Acad. Sci. USA, 42:43-47, 1956.

[21] B.W. Silverman. Density Estimation for Statistics and Data Analysis. Chapman and Hall, London, 1986.

[22] L.T. Tran. Kernel density estimation on random fields. J. Multivariate Anal., 34:37-53, 1990.

[23] L.T. Tran and Yakowitz S. Nearest neighbor estimators for random fields. $J$. Multivariate Anal., 44:23-46, 1993.

[24] W.B. Wu and J. Mielniczuk. Kernel density estimation for linear processes. Ann. Statist., 30:1441-1459, 2002.

[25] Q. Yao. Exponential inequalities for spatial processes and uniform convergence rates for density estimation. In Development of Modern Statistics and Related Topics - In Celebration of Prof. Yaoting Zhang's 70th Birthday, H. Zhang and J. Huang (edit.), World Scientific, Singapore, pages 118-128, 2003. 